\title{
Efficient use of paired spectrum bands through TDD small cell deployments
}

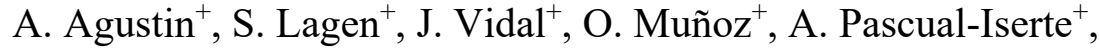 \\ G. Zhiheng*, W. Ronghui* \\ $\left(^{+}\right)$Signal Processing and Communications Group (SPCOM), Universitat Politècnica de \\ Catalunya, Barcelona, Spain \\ (*) Huawei Technologies Co., Ltd, Beijing, P.R. China \\ \{adrian.agustin, sandra.lagen, josep.vidal,olga.munoz, antonio.pascual\}@upc.edu \\ \{guozhiheng, wenronghui\}@huawei.com
}

\begin{abstract}
Traditionally, wireless cellular systems have been designed to operate in frequency division duplexing (FDD) paired bands that allocate the same amount of spectrum for both downlink (DL) and uplink (UL) communications. Such design is very convenient under symmetric DL/UL traffic conditions, as it used to be the case when voice transmission was predominant. However, due to the overwhelming advent of data services, which involves large asymmetries between DL and UL, the conventional FDD solution becomes inefficient. In this regard, flexible duplexing concepts aim to derive procedures to improve spectrum utilization by adjusting resources to actual traffic demand. In this work, we review these concepts and propose the introduction of time division duplexing (TDD) small eNBs (SeNB) to operate in the unused resources of an FDD-based system. This proposal alleviates the saturated DL/UL transmission commonly found in FDD-based systems through user offloading towards a TDD system based on SeNBs. In this context, the flexible duplexing concept is analyzed from three points of view: a) regulation, b) long term evolution (LTE) standardization, and c) technical solutions.
\end{abstract}




\section{Introduction}

The most salient feature in the evolution of mobile services is the imposition of data services over voice traffic demand, thus, requiring the redefinition of current wireless cellular networks and communication standards. Second and third generation wireless cellular systems were designed under a symmetric traffic assumption as a result of the predominance of voice traffic. Accordingly, the common technical solution adopted worldwide was the use of paired bands under frequency division duplexing (FDD). The legacy of this assumption has survived in fourth generation $(4 \mathrm{G})$ systems, even though a time division duplexing (TDD) frame definition was also early defined.

The new habits of users have produced high asymmetries in data traffic demand, i.e. the amount of data transmitted in the downlink (DL) connection is usually much larger than the amount of data in the uplink (UL) transmission [1]. The most conservative measured DL:UL traffic asymmetry ratio across different macro eNBs (MeNBs) is 4:1 [2] due to video downloading and internet browsing. On its turn, the uploading of shared contents in social media is generating the opposite tendency, attaining ratios of 1:4 [3]. Such time/space-varying unbalance of data traffic negatively affects the spectral efficiency of FDD-based systems since its inflexibility translates into an underuse of one band while the other band may be congested. This inefficiency could be reduced by adopting unpaired band technologies based on TDD, in which the use of radio resources dedicated to DL and UL transmissions can be flexibly adapted as a function of the actual traffic demand.

The present work explores how the spectral efficiency of long term evolution (LTE) FDD-based systems can be improved under traffic asymmetries by means of the flexible duplexing concept [4]. In particular, the proposed solution assumes an FDD MeNB serving area in which TDDbased small eNBs (SeNB) are deployed and operate in the unused resources. With the objective of having a clear idea of the benefits of the proposed solution, a simple scenario with one SeNB is considered, as it is shown in Figure 1. In this context, the following challenges have to be faced:

- Coexistence of adjacent FDD/TDD systems. Because of non-ideal transmit filters, adjacent channel interference (ACI) is originated from systems operating in adjacent bands. ACI can be managed either by imposing a minimum distance between transmitting nodes [5] or by defining a set of guard bands and power spectrum masks [6].

- Impact of different TDD-LTE frame pattern configurations. Conventionally, in TDD mode, all eNBs transmit simultaneously in DL and, at a different time instant, all UEs transmit in UL. This approach aims at limiting the active nodes that generate interference in each case. However, the use of flexible TDD MeNB/SeNBs entails a dynamic decision of their own TDD DL-UL frame pattern that introduces new types of interference in cellular systems, i.e. MeNBs/SeNBs can be interfered by other MeNBs/SeNBs. Nevertheless, if this interference is properly managed, significant throughput gains can be obtained in low-to-medium system loads [5].

- Shared spectrum access. Interference management is important when eNBs with different maximum transmitting power levels operate in the same radio resources. However, deploying outdoor SeNBs (with a maximum equivalent isotropical radiated power (EIRP) of $30 \mathrm{dBm}$ and a height below 12 meters) is a simple solution that reduces interference and allows a large reuse of the spectrum [7]. 
The proposed solution in this work has also been investigated in [8] where TDD cognitive SeNBs are allowed to exploit the FDD-DL spectrum. In that case, the TDD SeNB listens to the FDD UL signals in order to detect if there are active FDD-UEs (or MUEs in the following) in the neighboring area. If this is not the case, the UEs associated to the TDD SeNB (SUEs in what follows), and of course the SeNB itself, are allowed to transmit in the FDD-DL band. Another implementation of a TDD system in the unused FDD-UL spectrum is proposed also in [9], where the interference between the FDD-UL and TDD systems is avoided thanks to a tight time coordination between FDD and TDD systems.

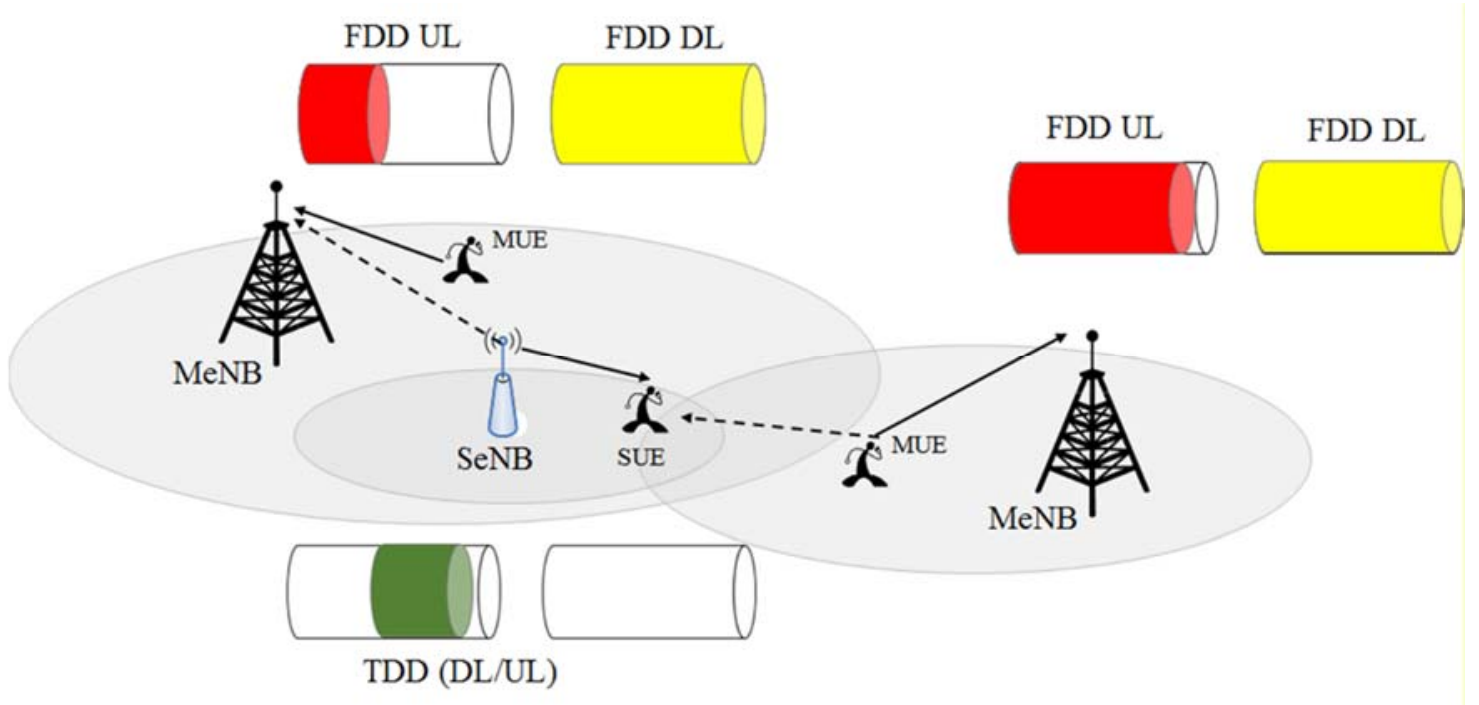

Figure 1. Example of the flexible duplex concept. There is one macro-cell area with unused resources in the UL but saturated in the DL. A TDD SeNB is allowed to operate in the unused resources while coexisting with the MeNB.

Introducing the flexible duplex concept by deploying multiple TDD SeNBs in wireless FDDbased communications systems entails dealing with many issues that are not currently found in current cellular wireless systems, in which SeNBs and MeNBs are assumed to operate either in different spectrum bands or, in case of coexisting in the same band, to use the same duplexing mode. With the objective of elucidating how the proposed approach impacts on system performance, a simplified scenario is analyzed here, where just a single TDD SeNB in the macrocell area adopts the flexible duplex concept, see Figure 1. How the position of the SeNB and its transmitted power impact on system performance and which FDD-band can be reused (UL or DL) are important aspects that need to be understood. This knowledge will be decisive to determine which of the existing TDD SeNBs deployed in the macrocell area can operate in the underutilized spectrum.

This paper examines alternatives and challenges in the implementation of the flexible duplexing concept in LTE. Specifically, Section 2 details the proposed schemes along with the pros and cons of reusing either the FDD-UL or the FDD-DL bands. Section 3 addresses the limitations of applying the flexible duplexing concept as a result of the application of current regulation and/or LTE standard constraints. Section 4 reviews future research lines related to multiple flexible duplex SeNBs. Finally, Section 5 underlines some conclusions. 


\section{Flexible use of the paired band}

In an FDD-based system, a guard band (usually of several MHz) is required to separate the paired $\mathrm{UL}$ and DL bands (usually a few MHz). Therefore, the current underutilized spectrum cannot accommodate a new FDD-based system. Fortunately, TDD-based systems are not affected by such guard band constraint. Accordingly, we investigate the deployment of a TDD SeNB operating in the unused spectrum of an FDD-based system. The proposed schemes for multiplexing MeNB and SeNB are described in Section 2.1. Since it is important to know how many resources are needed by the MeNB as a function of the traffic demand, Section 2.2 discusses how the resource provisioning could be estimated. Finally, the benefits in terms of user throughput and resource utilization of the proposed flexible duplexing concept are presented in Section 2.3.

\subsection{Flexible duplexing methods in LTE}

The options to implement the flexible duplexing concept depend on whether it is possible to release part of the licensed FDD spectrum or not, paying special attention to those frequency resources in LTE reserved for control channels. The first option, referred in the following as efficient in-band orthogonal use of the licensed spectrum, subsumes the case where the SeNB works in the band of interest through an orthogonal access manner. The different implementations are depicted in Figure 2: A) orthogonal in time, B) orthogonal in frequency (both cases are described in detail in Section 2.1.1). The second option, addressed in Section 2.1.2 and illustrated in Figure 2-C), assumes that the MeNB can make good use of the intra-band component carrier (CC) aggregation LTE feature, adapting its operational bandwidth according to the MeNB traffic demand. MeNB and SeNB work orthogonally in frequency but, in contrast to the approach in Figure 2-B), the FDD-based system can adapt its reserved resources and the SeNB can access the channel through frequency multiple access.

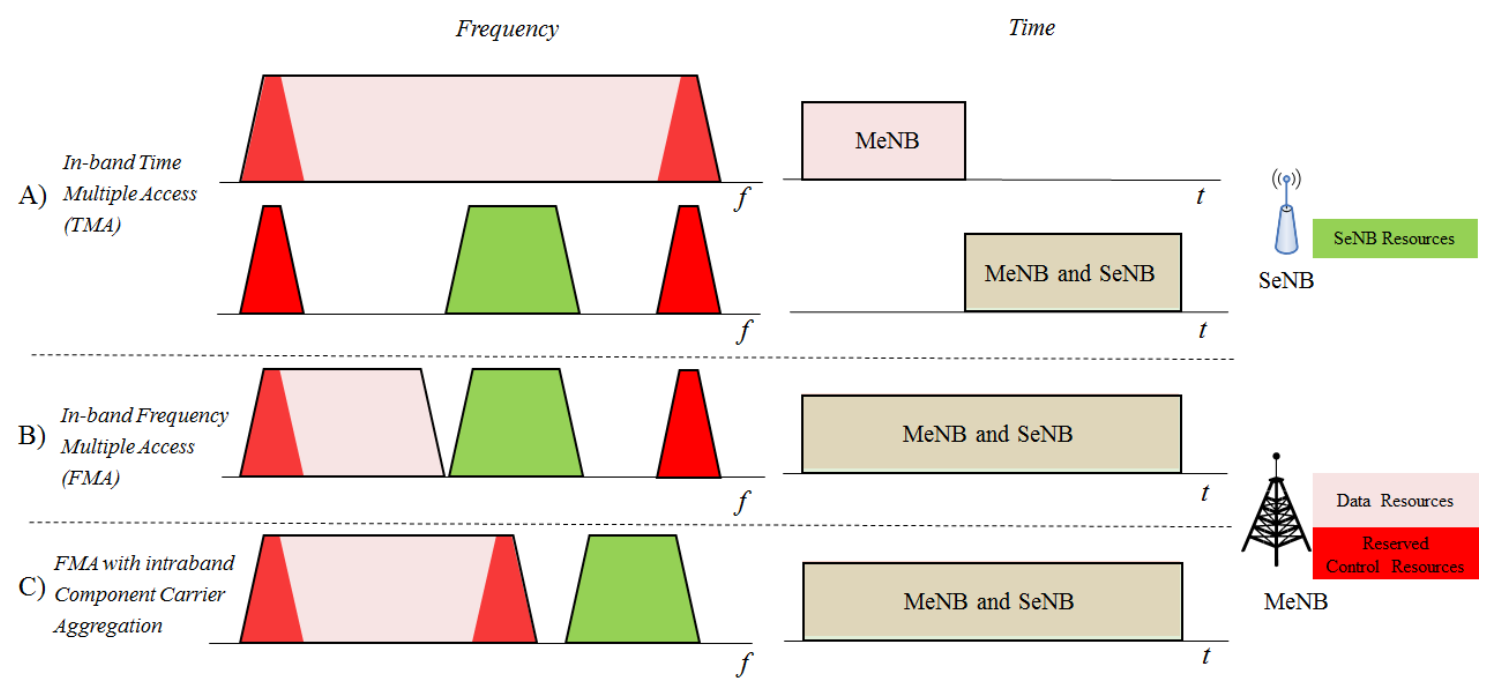

Figure 2. A), B) An overlaid TDD SeNB operates in the unused resources of FDD-UL under time multiple access and frequency multiple access, respectively. In C), the MeNB adjusts its operational

LTE FDD-UL bandwidth using CC aggregation and the TDD SeNB works in the released resources. Dark-red resources at the left-had side denote reserved frequencies for FDD controlplane communications. 


\subsubsection{Efficient in-band orthogonal use of the licensed spectrum}

SeNB and MeNB operate in the same band through orthogonal multiple access. The SeNBs might operate at the unused MeNB band in time-multiplexing access (TMA) or frequency-multiplexing access (FMA) (see Figure 2-A) and Figure 2-B) when FDD-UL band is reused). The second approach depicted in Figure 2-B) requires that the receiver at the MeNB has to be equipped with additional analog filters to avoid the undesired interference from the sidelobe signal transmission in adjacent bands coming from SeNBs. This is due to the fact that the bandwidth of the receive filter at MeNB corresponds to the whole operational bandwidth. Therefore, this option comes at the cost of losing flexibility since unused time frequency resources of the FDD band have to be identified beforehand and have to be fixed over time because analog filters are necessarily nonflexible.

Reusing the FDD-UL band with the in-band TMA approach demands paying special attention to physical UL control channels (PUCCH) allocated at the edge of the band (see dark-red resources in Figure 2). Those resources are used by MUEs to transmit acknowledgements at a predetermined delay after DL transmission. Therefore, SeNB and MeNB should agree on accessing those resources at different time instances, or SeNB and MeNB should be equipped with additional analog filters to preserve the adequate reception of PUCCH at the MeNB. Similarly, the in-band FMA needs that transmitting and receiving nodes are equipped with analog filters (see Figure 2B)) and in-band interference becomes ACI.

Reusing the FDD-DL band is more complicated due to the current frame structure defined by LTE. The reserved frequency resources for control-plane communications are found in the central part of the FDD-DL band, which complicates the adoption of the in-band FMA approach. Regarding the TMA approach, it is even worse because LTE defines several DL subframes for transmitting system information, which leaves just two subframes (out of ten) for the TDD SeNB, as it will be detailed in Section 3.2.

\subsubsection{Efficient use of the CC-based licensed spectrum}

LTE allows reconfiguring bands thanks to the CC aggregation concept [10]. Here, it is assumed that the licensed spectrum is divided in CCs, and there is an entity responsible for the dynamic long/medium-term resource allocation that selects the number of required CCs by the MeNB as a function of the traffic demand. In such scenario, the unused CCs can be engaged by the TDD SeNB.

Using this approach, the FDD MeNB and the TDD SeNB operate on orthogonal carriers, which are isolated thanks to the respective analog transmit filters (see Figure 2-C)). The source of interference is ACI between both systems: i) TDD SeNB (in DL) over the FDD MeNB when the FDD-UL spectrum is reused, and ii) FDD MeNB over the TDD SeNB (in UL) when the FDDDL spectrum is reused. In all cases, the interference level depends on the probability of line-ofslight (LOS) between MeNB and SeNB, but this can be reduced by deploying SeNBs at a lower height than MeNBs. 


\subsection{Resource provisioning}

The use of the flexible duplexing concept requires a tight estimation of the amount of spectrum that will not be employed by the FDD MeNB system. A suitable key performance indicator (KPI) that contains this information is the resource utilization (RU). This metric reports the ratio between the total number of resources used by data traffic over the total number of resources available for data traffic. More explicitly, the RU of the $k$-th cell in the $d$-th transmit direction (either DL or UL) $\left(\rho_{k}^{d}\right)$ can be estimated based on the traffic statistics and the average spectral efficiency as [11]:

$$
\rho_{k}^{d}=\frac{\text { average offered traffic }}{\text { traffic served }}=\frac{\lambda_{k}^{d} L_{k}^{d}}{x_{k}^{d}} \frac{1}{C_{k}^{d}},
$$

where $\lambda_{k}^{d}$ is the mean packet arrival rate (in packets/s), $L_{k}^{d}$ denotes the mean packet length (in bits/packet), $x_{k}^{d}$ refers to the number of resources, and $C_{k}^{d}$ is the average spectral efficiency of the $k$-th cell in the $d$-th transmit direction (in bits/s/resource). Furthermore, the average number of bits in the queue of the $k$-th cell in the $d$-th transmit direction $\left(W_{k}^{d}\right)$ can be modeled as a function of the RU when packet arrival instants follow a Poisson process. In such a case, for $\rho_{k}^{d} \leq 1$, we have the following expression [11]:

$$
W_{k}^{d}=\frac{\rho_{k}^{d}}{1-\rho_{k}^{d}} \frac{\sigma_{k}^{2 d}+\left(L_{k}^{d}\right)^{2}}{2 L_{k}^{d}},
$$

where $\sigma_{k}^{2 d}$ denotes the variance of the packet length. Note that a large RU factor implies a large average queue size.

From expressions (11) and (22) one can either estimate how many resources are needed as a function of the traffic statistics or derive a maximum RU for a given target $W_{k}^{d}$, respectively. This information is useful to define the operation bandwidths of MeNB and SeNB from traffic statistics.

\subsection{Performance evaluation}

In this section, we present some simulation results for the access methods described in Section 2.1. The scenario consists of one FDD MeNB and one TDD SeNB, which operates at the unused portion of the FDD spectrum. Both are separated by a hundred meters. The complete scenario and simulation assumptions are included in Table 1.

The following techniques are evaluated:

- $\quad F M A$ (in-band FMA and CC-based FMA): FMA with frequency multiplexing between FDD MeNB and TDD SeNB, including ACI,

- $\quad$ TMA (in-band TMA): TMA with time multiplexing between MeNB and SeNB, including 1 guard subframe,

- only MeNB: one MeNB operating in the paired spectrum.

Data traffic follows a Poisson process with packet arrival rates $\lambda_{D L}$ and $\lambda_{U L}$ (in packets/s) for DL and UL transmissions, respectively. Two different traffic asymmetries are considered: 
- $\quad \lambda_{D L}=10 \times \lambda_{U L}$ : DL traffic is 10 times larger than UL traffic, originating unused resources in the FDD-UL band.

- $\quad \lambda_{U L}=10 \times \lambda_{D L}: \mathrm{UL}$ traffic is 10 times larger than DL traffic, originating unused resources in the FDD-DL band.

For each case, two packet arrival rates are evaluated: $\lambda_{D L}=\{1,1.5\}$ packets/s for $\lambda_{D L}=10 \times \lambda_{U L}$ and $\lambda_{U L}=\{1,1.5\}$ packets/s for $\lambda_{U L}=10 \times \lambda_{D L}$ (emulating medium and high traffic loads).

By taking into account the values of the packet arrival rates for each traffic asymmetry, the measured RU at the FDD MeNB (according to (11)) is less than 30\% in the FDD-UL band (under $\lambda_{D L}=10 \times \lambda_{U L}$ traffic asymmetry) and less than $14 \%$ in the FDD-DL band (under $\lambda_{U L}=10 \times \lambda_{D L}$ traffic asymmetry). The TDD SeNB operates in the unused resources in the following way:

- FMA: In FDD-UL band reuse, SeNB operates over $7 \mathrm{MHz}$ bandwidth with a TDD duplexing ratio 7:3 (7 DL and 3 UL subframes). In FDD-DL band reuse, the SeNB operates over $8.7 \mathrm{MHz}$ bandwidth with a TDD duplexing ratio 1:9.

- TMA: It is just evaluated for FDD-UL band reuse due to the reasons provided in Section 2.1.1 and 3.2. The SeNB operates over the whole spectrum but only $60 \%$ of the time, with a TDD duplexing ratio $4: 2$.

\begin{tabular}{|l|l|}
\hline \multicolumn{2}{|c|}{ General system parameters } \\
\hline MeNBs deployment & $\begin{array}{l}\text { One sector of a macrocell area with 1 FDD MeNB. Hexagonal deployment of FDD } \\
\text { MeNBs. Two interfering MeNBs operate in FDD with normal usage at Inter-site } \\
\text { distance of 500 m }\end{array}$ \\
\hline SeNB deployment & 1 TDD SeNB at a distance 100 m from MeNB. \\
\hline UEs deployment & 50 UEs uniformly distributed within the macrocell area \\
\hline Spectrum & Licensed paired FDD: 10 MHz for DL and 10 MHz for UL \\
\hline Frequency carrier & $2.5 \mathrm{GHz}$ \\
\hline Transmit power & $46 \mathrm{dBm}$ (MeNB), 24 dBm (SeNB), 23 dBm (UE) \\
\hline MeNB antenna system & $17 \mathrm{dBi}, 3 \mathrm{D}$, Sectorized, 2 antennas \\
\hline SeNB antenna system & $5 \mathrm{dBi}, 2 \mathrm{D}$, Omnidirectional, 2 antennas \\
\hline UE antenna system & $0 \mathrm{dBi}, 2 \mathrm{D}$, Omnidirectional, 2 antennas \\
\hline Noise figure & $5 \mathrm{~dB}$ at MeNB and SeNB, $9 \mathrm{~dB}$ at UEs \\
\hline Noise spectral density & $-174 \mathrm{dBm} / \mathrm{Hz}$ \\
\hline Propagation conditions & $\begin{array}{l}\text { Pathloss and shadowing as in [5]. Frequency selective fading follows the typical } \\
\text { urban model. }\end{array}$ \\
\hline Cell association & $\begin{array}{l}\text { UEs are associated to the MeNB or SeNB using the reference signal received power } \\
(\mathrm{RSRP}) \text { combined with a cell range expansion (CRE) bias. CRE is adjusted so as to } \\
\text { get approximately 10 UEs associated to SeNB }\end{array}$ \\
\hline Traffic model & FTP model 3. Packet size 2 Mbits. \\
\hline
\end{tabular}

Table 1. Simulation assumptions.

Two metrics are considered as performance indicators: a) the RU presented in (11), measured as the average number of resource blocks needed for the communication over the total number of available resource blocks, and b) the mean of the user throughput (UT) in Mbits/s. 
The following figures show the mean UT versus the RU of the MeNB and SeNB for the two considered traffic asymmetries ( $\lambda_{D L}=10 \times \lambda_{U L}$ in Figure 3 and $\lambda_{U L}=10 \times \lambda_{D L}$ in Figure 4). Several conclusions can be drawn.

When there are unused resources in the FDD-UL band and the ones in the FDD-DL band are nearly saturated (see Figure 3):

- An improvement of 'UT DL' at both the MeNB and the SeNB is obtained with FMA and TMA as compared to the case of having only the MeNB active since more DL traffic can be served. The relative gains are up to 7\% in terms of 'UT DL' both at MeNB and SeNB for $\lambda_{D L}=1$ packets/s. Nevertheless, when the traffic increases up to $\lambda_{D L}=1.5$ packets $/ \mathrm{s}$, 'UT DL' relative gains significantly improve: $20 \%$ at MeNB and $49 \%$ at SeNB (see Figure 3right).

- For medium traffic loads ( $\lambda_{D L}=1$ packets/s in Figure 3-left), thanks to the reuse of the licensed bandwidth for UL with FMA and TMA, the MeNB DL reduces its RU as compared to the case of having only the MeNB active. So, MeNB is decongested.

- The ACI (SeNB to MeNB and SUE to MeNB) in FMA imposes a lower transmission rate, so that more resources are needed (see 'RU DL SeNB', 'RU UL MeNB', and 'RU UL SeNB'). For example, the 'RU DL SeNB' is 0.37 with FMA and 0.28 with TMA for $\lambda_{D L}=1.5$. The UT becomes lightly degraded with FMA at high traffic loads due to the activity of the SeNB. It generates ACI towards the MeNB, so the transmission rate of MUEs is lowered, but being active more time, and negatively impacting on the SeNB when it is in UL (see 'UT UL' in Figure 3-right). This effect is avoided with TMA. 'UT UL' at MeNB and SeNB is 0.36 and $0.35 \mathrm{Mbits} / \mathrm{s}$ with FMA, respectively, while it takes values of 0.38 and $0.37 \mathrm{Mbits} / \mathrm{s}$ at MeNB and SeNB, respectively, under TMA.
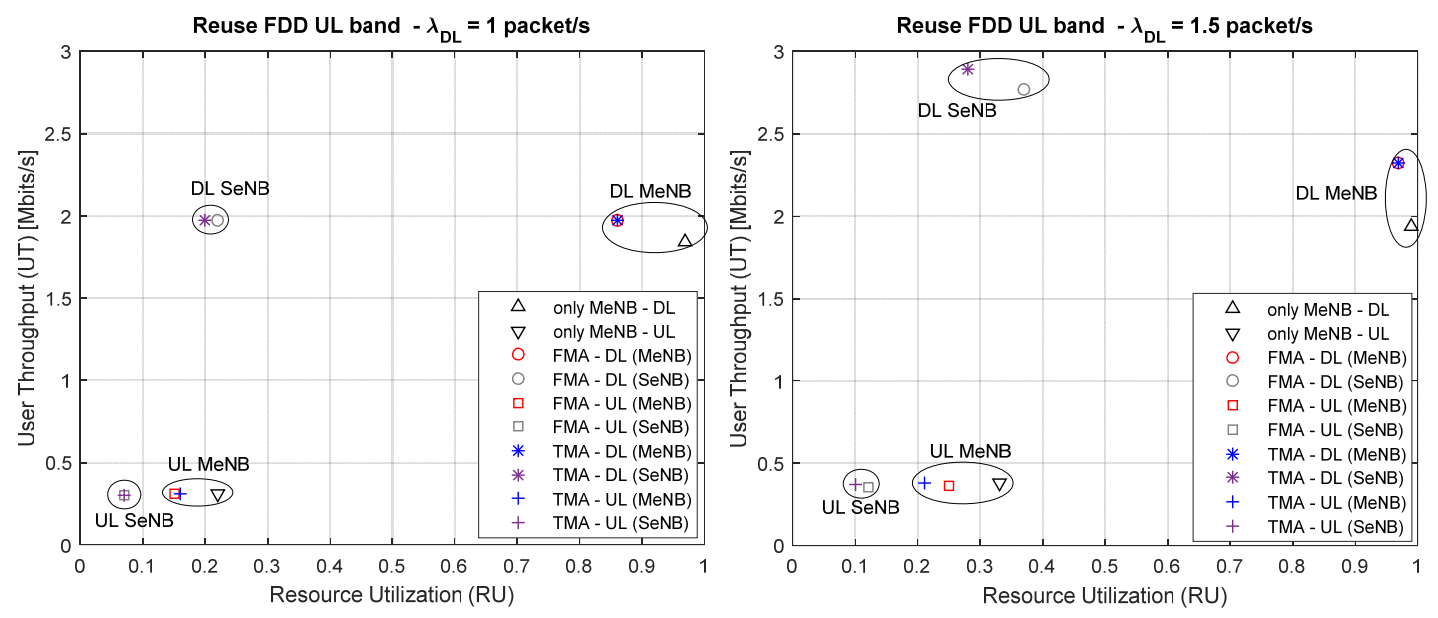

Figure 3. Mean UT (in Mbits/s) versus RU for FDD-UL band with $d=100 \mathrm{~m}$. Left) $\lambda_{D L}=1$, Right) $\lambda_{D L}=1.5$ (packets/s). Traffic asymmetry: $\lambda_{D L}=10 \times \lambda_{U L}$. 

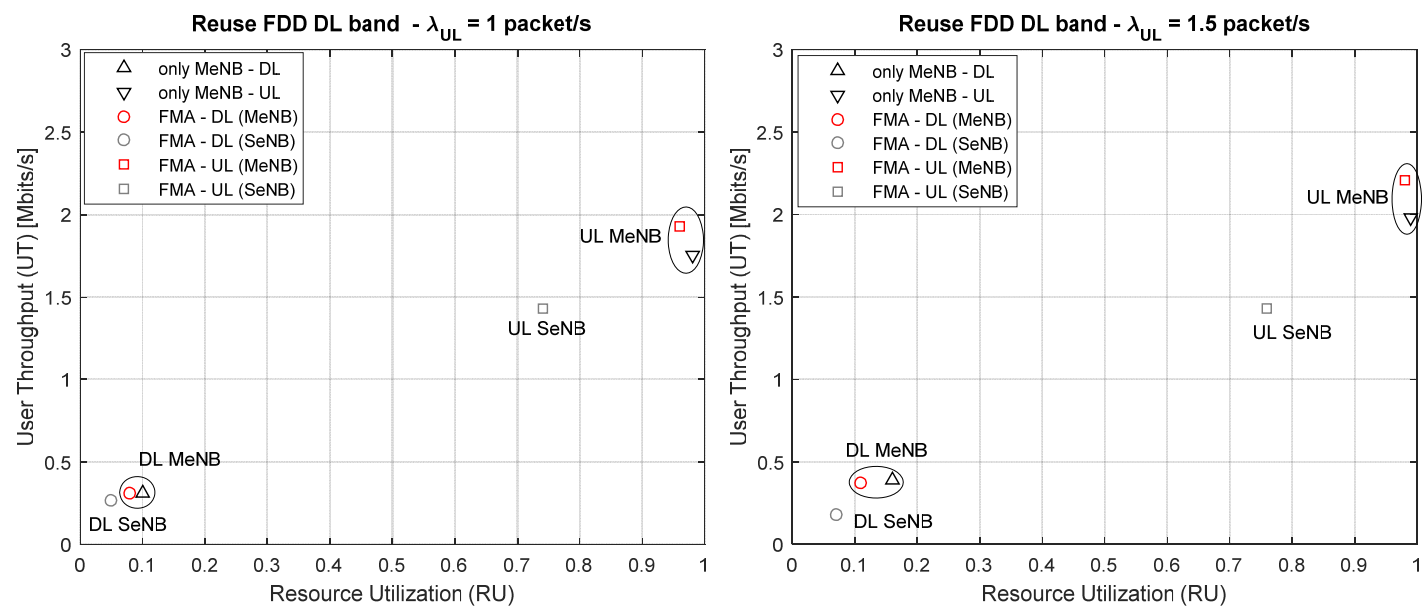

Figure 4. Mean UT (in Mbits/s) versus RU for FDD-DL band reuse with $\boldsymbol{d}=100 \mathrm{~m}$. Left) $\lambda_{U L}=1$, Right) $\lambda_{U L}=1.5$ (packets/s). Traffic asymmetry: $\lambda_{U L}=10 \times \lambda_{D L}$.

On the other hand, when the underutilized resources are in the FDD-DL band, results are affected by two main impairments: a) DL interference from neighbouring MeNBs (co-channel external interference), which can significantly degrade the system performance since the MeNB interferes with the SeNB transmissions both in DL and UL, and b) $A C I$ at $S e N B$, which is important because transmitters in the neighbouring band are MeNBs. From Figure 4 we infer that:

- An improvement of the 'UT UL MeNB' is obtained as compared to the case of having only the MeNB active, as more UL traffic can be served. The relative gains are: $10 \%$ for $\lambda_{D L}=1$ packets $/ \mathrm{s}$ and $12 \%$ for $\lambda_{D L}=1.5$ packets $/ \mathrm{s}$.

- For the two packet arrival rates, the 'UT UL SeNB' is significantly degraded with respect to the 'UT UL MeNB'. The relative losses are of $19 \%$ for $\lambda_{D L}=1$ packets $/ \mathrm{s}$ and of $28 \%$ for $\lambda_{D L}=1.5$ packets $/ \mathrm{s}$. This is due to co-channel external interference from neighbouring MeNBs, which significantly impacts on 'UT UL SeNB'. In addition, the effect of cochannel external interference also degrades 'UT DL SeNB'.

- There is nearly no impact on 'UT DL MeNB' when reusing the FDD-DL band and on 'UT UL MeNB' when reusing the FDD-UL band, and similar values are obtained as compared to the case of having only the MeNB, since all the traffic that arrives at the system is being served. According to equation (22), low resource utilization denotes an small average queue size in the system.

\section{Current limitations}

In spite of the promising benefits shown by the flexible duplexing concept in Section 2.3, its implementation in the short term must face several challenges from the point of view of regulation and LTE standardization.

\subsection{Regulation}

Radio spectrum regulators define which type of transmissions are allowed on different parts of the spectrum. In general, the FDD-UL spectrum can be employed by mobile stations or end-users, but not by base stations. In this regard, a survey to different regulators revealed that at least in US 
the flexible duplex concept is allowed in the band 1719-1755 MHz, [12]. On the other hand, in Europe (ECC PT1) or Japan (ARIB), the flexible use of UL and DL for FDD bands is not allowed. Nevertheless, the use of SeNBs with a transmit power equivalent to the maximum allowed in the UL by regulation would satisfy all technical requirements. In this regard, ECC PT1 is open to receive new results about the benefits of the flexible use of the licensed spectrum bandwidth.

\subsection{LTE compliance}

The following aspects limit the definition of LTE standard-compliant procedures for the implementation of the flexible duplexing concept.

Operating band definition. LTE defines a set of operating bands along with its use: FDD (bands 1-32) or TDD (bands 33-44), see [10]. From the comparison of UL-DL FDD bands and TDD bands (see Figure 5), we can observe that, with the current definition, not all FDD bands could be reused by TDD systems. However, the flexible duplexing concept might be considered in Europe and Hong Kong because band 7 is actually in use, so that TDD SeNBs operating in band 41 could reuse the underutilized spectrum. The UEs in the area should just measure the control channels of MeNB and SeNB, and decide their association to one of them.

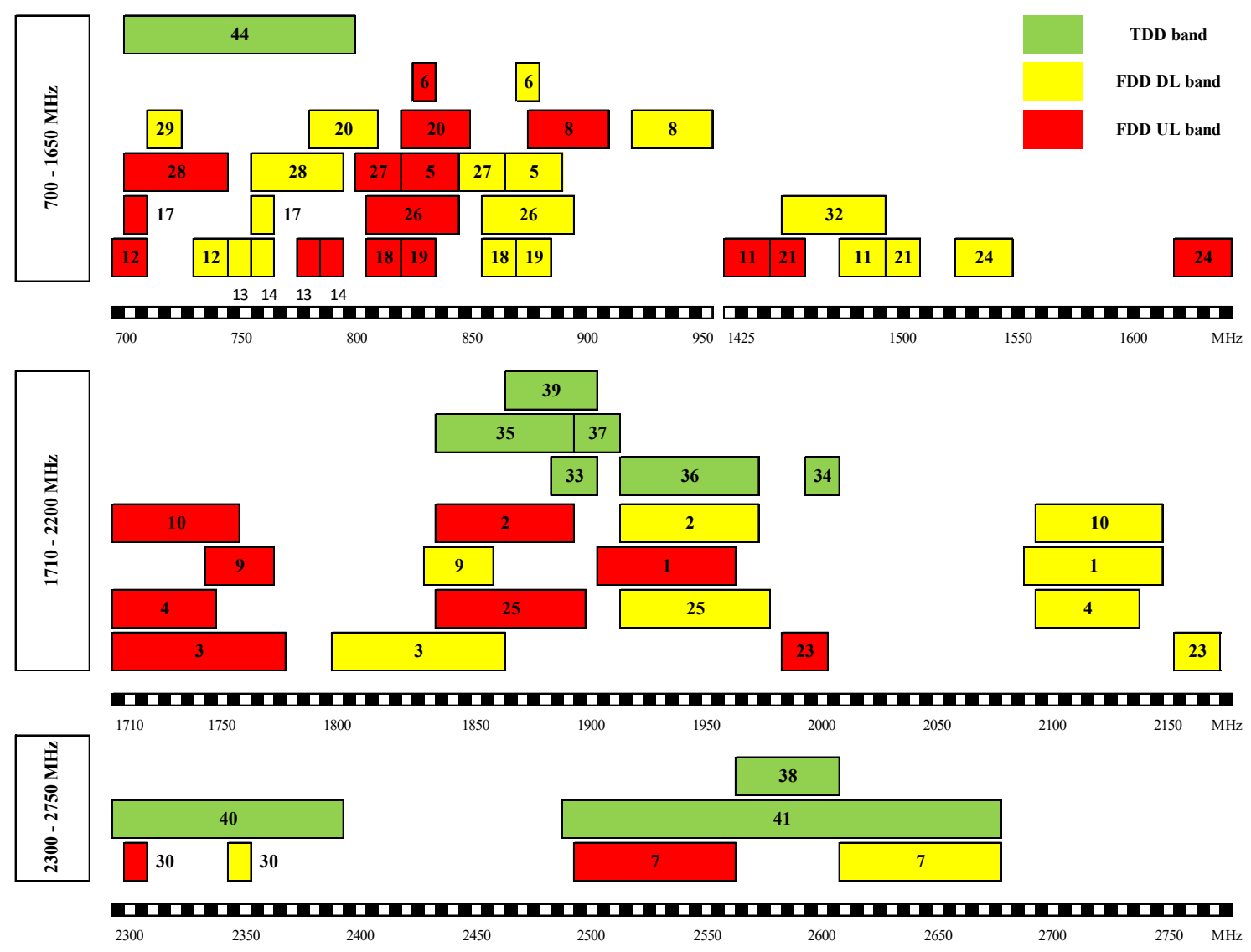

Figure 5. E-UTRA FDD (red and yellow) and TDD (green) operating bands. Proposed flexible duplexing methods would be standard-compliant in those bands where TDD and FDD overlap. 
PUCCH in the FDD UL band. The current design of LTE FDD systems places PUCCH in the resource blocks located at the edge of the band [13]. Those resources are devoted to transmit system information of UE. This system constraint limits the flexibility for seizing the unused time-frequency resources in-band TMA approach as described in Section 2.1.1.

Frame structure in FDD DL band. Even in situations of low DL traffic, the information necessary to operate the system (to be more specific, synchronization signals and system information and paging) needs to be transmitted by the FDD-DL cell so that terminals can detect and connect to a cell. In FDD, the subframes where such information is provided are subframes $0,4,5$, and 9 within an LTE frame composed of 10 subframes. Therefore, these subframes must be used by the FDD cell and cannot be used by a TDD cell. Similarly, the TDD SeNB needs also to transmit the information necessary to operate the system. In TDD, such information is provided through subframes 0,1 (special subframe), 5, and 6 (special subframe) within a frame of 10 subframes. It turns out that only 2 subframes could be reused for data transmission with the in-band TMA approach. On the other hand, the SeNB should be deployed in a narrower bandwidth placed at one side of the band for the in-band FMA approach because all synchronization signals of FDDDL occupy the central part of the band.

Carrier Aggregation. Currently, 3GPP standard does not allow tackling situations where the traffic asymmetry is higher in the FDD-UL band because, by definition, the CCs in the UL smaller than in the DL [13]. This feature does not allow extending the concept explained in Section 2.1.2 to re-use the underutilized FDD spectrum in the FDD-DL band.

\section{Future challenges and research}

Future research encompasses three different research lines: $i$ ) the management of the operation of multiple SeNBs in the unused spectrum for the multiplexing methods explained in Section 2, ii) the study of opportunistic multiple access (OMA) methods, and iii) the design of advanced interference cancellation receivers for OMA. One of these research lines presents important technical challenges that require further investigation for the deployment of the flexible duplex concept.

Multiple SeNBs. Resources among SeNBs should be distributed by taking into account the actual traffic demand per SeNB. The RU presented in Section 2.2, in addition to allowing the identification of the required spectrum, is a useful metric to derive resource provisioning schemes in a multi-cell scenario (e.g. multiple TDD SeNBs that exploit the spectrum released by the FDD MeNB). An efficient resource provisioning should distribute resources among cells in a balanced way while trying to avoid very different occupancies. In this sense, a meaningful optimization criterion is the minimization of the maximum RU among cells, so that resources are fairly distributed and more resources are given to those cells with larger traffic loads and/or those cells experiencing greater delays. For example, long-term graph colouring-based resource provisioning schemes are presented in [15] with the objective of optimizing the RU factors of multiple TDD SeNBs when either orthogonal access is assumed or reuse of resources among non-interfering SeNBs is considered.

OMA in OFDM-based systems. Allowing that a SeNB works in part of the spectrum of the MeNB in an opportunistically way (i.e. non-orthogonal) demands a tight synchronization (timing offset adjustments) so as to maintain the orthogonality of OFDM carriers at SeNB and MeNB. For example, in the heterogeneous scenario described in [14], which consists of one MeNB and one 
SeNB both sharing band and duplexing, it was shown that UEs have to advance their UL transmissions (pre-compensation) not only by taking into account the propagation delay with the SeNB but also by considering the propagation delay with the MeNB and the received frame boundary. The cyclic prefix in OFDM systems combats this issue, in addition to maintaining the orthogonality among subcarriers. However, in the flexible duplexing concept, SeNBs work in TDD while MeNB is FDD-UL, which means that synchronization is more challenging because the SeNB DL transmission should be pre-compensated by taking into account the neighbouring FDD MeNBs and the TDD SeNB working in UL.

Advanced interference canceller in OMA. If signal time offsets are properly pre-compensated to avoid the time misalignments with a FDD MeNB, then OMA can be used by the TDD SeNBs. The interference received by the MeNB from the transmitting SeNBs might be very high due to LOS condition in the MeNB-SeNB link. A successive interference canceller (SIC) would alleviate the effect of interference, but the performance of SIC will depend on the received interference level (distance between MeNB-SeNB) and the bit rate selected by the SeNB (DL transmissions to a SUE).

\section{Conclusions}

The flexible duplexing concept allows for improvements in the system efficiency of paired-based systems by using TDD SeNBs. When allowing a TDD SeNB to operate in the underutilized FDDUL band, we have observed that the DL user throughput is improved and the congestion of the MeNB is reduced. The effect of ACI for the FMA schemes becomes relevant when: a) the SeNB is close to the MeNB, b) the SeNB transmits with high power, and c) the activity of UEs and the SeNB is high. On the other hand, when a TDD SeNB reuses the underutilized FDD-DL band, the potential gains are reduced because of the external interference coming from neighbouring MeNBs that are transmitting DL signals. The benefits of the flexible duplexing concept can be enlarged when combined with the deployment of multiple SeNBs and the application of interference management techniques, but its actual implementability is also tied to the limitations imposed by regulations and standards.

\section{Acknowledgments}

This work has been supported by Huawei Technologies Co., Ltd. and by the "Ministerio de Economía, Industria y Competitividad" of the Spanish Government and European Regional Development Fund (ERDF) TEC2013-41315-R DISNET and "Agencia Estatal de Investigación" and ERDF TEC2016-77148-C2-1-R 5G\&B-RUNNER-UPC

\section{References}

DIGITALEUROPE: Call for Timely Harmonisation of the $1452-1492 \mathrm{MHz}$ and $2300-2400 \mathrm{MHz}$ Bands to Support Delivery of the EU Radio Spectrum Policy Programme Objectives, Brussels, 21st February 2012 

Globecom 2013 interference management and traffic adaptation". Release 11. June 2012

$[6]$,

ECC Report 119. Coexistence between Mobile Systems in the frequency band at the FDD/TDD Boundary. June 2008

Real Wireless. Low-power shared access to spectrum for mobile broadband. Ofcom project MC/073. March 2011

R. Berangi, S. Saleem, M. Faulkner, W. Ahmed, "TDD cognitive radio femtocell network (CRFN)", in Proc. IEEE Int. Symposium of Personal, Indoor and Mobile Radio Communications, Sep. 2011

3GPP. R1-134295, "FDD-TDD CA/Dual Connectivity solution exploiting traffic asymmetry in duplex-neutral bands" (Oct-2013).

Kumar, D. Manjunath, and J. Kuri, Communication Networking: an analytical approach. Morgan Kaufmann Publishers, Elsevier, 2004 3GPP TR 36.882, "Study on regulatory aspects for flexible duplex for E-UTRAN", Release 13, June 2015 Press, 2011

N. Himayat, et al., "Synchronization Uplink Transmissions from Femto AMS", IEEE C802.16m09/30775r2, January 2010

S. Lagen, O. Muñoz, A. Pascual-Iserte, J. Vidal, A. Agustin, "Long-term provisioning of radio resources based on their utilization in dense OFDMA networks", IEEE Int. Symp. on Personal, Indoor and Mobile Radio Communications., Valencia (Spain), Sep. 2016. 